\title{
Adsorption of Ellipsoidal Particles at Liquid-Liquid Interfaces
}

\section{Journal Article}

\section{Author(s):}

Coertjens, Stijn; de Dier, Raf; Moldenaers, Paula; Isa, Lucio; Vermant, Jan

Publication date:

2017-03

Permanent link:

https://doi.org/10.3929/ethz-b-000129874

\section{Rights / license:}

In Copyright - Non-Commercial Use Permitted

\section{Originally published in:}

Langmuir 33(11), https://doi.org/10.1021/acs.langmuir.6b03534 


\title{
Adsorption of ellipsoidal particles at liquid-liquid interfaces
}

\author{
Stijn Coertjens, ${ }^{\dagger}$ Raf De Dier, ${ }^{\ddagger}$ Paula Moldenaers, ${ }^{\dagger}$ Lucio Isa, $^{\top}$ and Jan \\ Vermant ${ }^{*, \ddagger}$ \\ $\dagger$ Department of Chemical Engineering, KU Leuven, B-3001 Leuven, Belgium \\ $\ddagger$ Department of Materials, ETH Zürich, CH-8093 Zürich, Switzerland \\ ILaboratory for Interfaces, Soft Matter and Assembly, Department of Materials, ETH \\ Zürich, CH-8093 Zürich, Switzerland \\ E-mail: jan.vermant@mat.ethz.ch
}

\begin{abstract}
The adsorption of particles at liquid-liquid interfaces is of great scientific and technological importance. In particular for non-spherical particles, the capillary forces which drive adsorption vary with position and orientation and complex adsorption pathways have been predicted by simulations. Based on the latter it has been suggested that the time scales of adsorption are determined by a balance between capillary and viscous forces. However, several recent experimental results point out the role of contact line pinning in the adsorption of particles to interfaces, and even suggest that the adsorption dynamics and pathways are completely determined by the latter, with the timescales of adsorption being determined solely by particle characteristics. In the present work the adsorption trajectories of model ellipsoidal particles are investigated experimentally using cryo-SEM and monitoring the altitudinal orientation angle using high speed confocal microscopy. By varying the viscosity and the viscosity jump across the interfaces we specifically interrogate the role of viscous forces.
\end{abstract}




\section{Introduction}

Particles at fluid-fluid interfaces are of great scientific interest ${ }^{1}$. The rules which control interaction forces for colloidal systems in bulk are no longer as simple or as subtle for particles at interfaces. For example, electrostatic and Van der Waals interactions are strongly enhanced $^{2,3}$ where the former can be used to direct the formation of non-close packed twodimensional crystals useful as model materials ${ }^{4-6}$. For rough or non-spherical particles strong lateral capillary interactions arise, due to the deformation of the interface caused by an undulating contact line or by particle shape effects ${ }^{7-9}$. However, the structures which are the result of these interactions can be exploited in many technological applications. Dense and flocculated 2D suspensions can be used to stabilize interfaces in foams ${ }^{10,11}$, PickeringRamsden emulsions ${ }^{12}$ or in so-called bijels ${ }^{13,14}$. 2D-colloidal crystals can be used in surface nano-patterning ${ }^{15}$. Furthermore, exploiting particle shape effects, for example by making the particles ellipsoidal in nature, leads to an even more spectacular stabilisation of interfaces in emulsions ${ }^{16}$, possible through strong effects on the interfacial rheology. Ellipsoidal particles also lead to a suppression of the infamous coffee ring effect in drying suspensions ${ }^{17,18}$.

There are several factors which come into play in all these applications, but an important one is the adsorption behaviour of particles at a fluid-fluid interface. There is a large reduction of free energy upon trapping a single particle at a liquid-liquid interface ${ }^{2,19,20}$. A single particle of radius $r$ sitting between oil (o) and water (w), and with a contact angle of $\theta$ reduces the energy with $\Delta E_{w}$

$$
\Delta E_{w}=\pi r^{2} \sigma_{o w}(1 \pm \cos \theta)^{2}
$$

where the sign is positive for the sphere to be moved into the oil phase and negative for

removal in the water phase. These energies can be very substantial. For example, a sphere of radius $r=1 \mu \mathrm{m}$ at an oil/water interface where $\sigma_{o w}=50 \mathrm{mN} / \mathrm{m}$ and a contact angle of 
$\theta=\pi / 2$ will require an energy of $50 \times 10^{6} k T$ to remove it. Such a large binding energy implies that particles in this size range are essentially irreversibly attached to the interface. The predictions of such a continuum theory even seem to hold down to the nanoscale ${ }^{21}$. This irreversible binding is unlike the one small surfactant molecules, which will continuously be exchanged with the bulk phases.

However, so far only few studies have focussed on the kinetics of adsorption of particles. For spherical particles, the adsorption energies and kinetics have been inferred from measurements of the interfacial tension using a pendant drop apparatus. Effects of particle size $^{21}$ and surface charge ${ }^{22}$ on the global adsorption kinetics have been investigated. Using holographic microscopy Kaz et al. monitored the adsorption of single particles and observed that the fast initial adsorption was followed by an intriguing and unexpectedly slow relaxation $^{23}$, which was been attributed to surface defects and contact line pinning ${ }^{24,25}$. Boniello and coworkers showed that a particle straddling an air/water interface feels a large viscous drag that is unexpectedly larger than the one measured in the bulk. They suggested that such a result arises from transient (thermally activated) fluctuations of the interface at the solid/air/liquid triple line and their coupling to the particle $\mathrm{drag}^{26}$, rather than invoking static heterogeneities as done in the work of Kaz et al. ${ }^{23}$. In congruence with such observations of important effects near the contact line, Coertjens et al. ${ }^{27}$ showed how surface heterogeneities influence the contact line shape, and consequently the wetting, of ellipsoids at a water-oil interface, and that these aspects become increasingly more important for ellipsoids with increasing aspect ratio.

Establishing the base case of adsorption of anisotropic particles to simple liquid-liquid interfaces is relevant for understanding their role in stabilizing emulsions ${ }^{16}$ and preventing the coffee stain effect in drying drops ${ }^{17}$, but also on how such particles interact with cell membranes ${ }^{28-30}$. Concerning ellipsoidal particles, mainly numerical simulation approaches 
have been used so far to understand the adsorption dynamics. De Graaf et al. ${ }^{31}$ used a triangular tessellation technique to calculate the adsorption trajectories (altitudinal orientation angle of the particles versus position with respect to the interface) for different initial orientations. The free energy landscape for an adsorbing particle is complex. De Graaf et al. predict that independent of the initial orientation, there is an attractor curve which predicts that the particle penetrates the interface orthogonal to the interface, rising above it until it reaches its maximum height above the interface and the rotating towards the interface to assume a planar configuration. However, the free energy method does not take into account interfacial deformation and colloidal interactions. Günther et al. ${ }^{32}$ used a more elaborate simulation, using a combined multicomponent lattice Boltzmann and molecular dynamics approach. Similar to De Graaf et al., adsorption trajectories are complicated and non-monotonic. An attractor exists which predicts the particle to penetrate the interface at an angle close to $90^{\circ}$. However, they do not predict that the particles rise above the interface, rather the rotation sets in when the particle reaches it middle point. In both simulations however, the pathway is complex and there is a link between the particle orientation and the height of the particle to the interface. Initially the orientation angle is constant and the particle penetrates the interface. In a second stage the particle rotates and reaches its in plane configuration. Despite the differences in the maximum height of the particle relative to the interface between the two approaches, the two stage process can be considered to be a hallmark of capillary adsorption. Taking into account capillary forces and viscous friction predict time scales in the range of microseconds to seconds, for typical viscosities and surface tensions $^{31}$.

Very recently, Wang et al. ${ }^{33}$ reported data using holographic microscopy experiments of adsorption of model non-spherical particles. Their results do not show the expected two stage process and the adsorption dynamics is shown to be much slower than predicted from capillarity. The pathway to equilibrium is observed to be simply continuous evolution of 
both vertical position and angle. Wang et al. ${ }^{33}$ argue, using a simple quasi-static model of contact line motion originally proposed to explain the slow, long-time, adsorption dynamics observed for spheres by Kaz et al. ${ }^{23}$, that the adsorption dynamics and pathways are completely determined by contact line pinning. However, the time resolution of the adsorption trajectories was limited, and an optical tweezer was needed to push the particles up to the interface which may influence the initial stages of adsorption and penetration. In addition to the slow kinetic evolution of the contact line, which previously was indirectly shown by cryo-SEM contact-angle measurements ${ }^{27}$, we argue that the capillary forces are still expected to dominate at short times and hence also viscous forces may still play a role at this point.

The present work aims at differentiating between the fast and slow mechanisms using high-speed confocal microscopy. We will experimentally measure the adsorption kinetics of a colloidal prolate ellipsoid at a planar liquid-liquid interfaces, using a combination of twoand three-dimensional high-speed confocal scanning laser microscopy and cryo-SEM. Model polystyrene ellipsoidal particles, for which the static wetting has been characterized ${ }^{27}$, with aspect ratios ranging from 4 to 6 will be used and weak buoyancy effects are used to get the particles to the interface. By varying the viscosity and the viscosity jump across the interfaces we hope interrogate the role of viscous forces, at least during the initial stages.

\section{Materials and Methods}

\subsection{Synthesis of charge-stabilized fluorescent polystyrene ellip- soids}

Ellipsoidal particles are produced by using the film- stretching method introduced by Ho et al. ${ }^{34}$, in which spherical PS particles are embedded in a polyvinylalcohol (PVA) matrix and heated above the melting temperature of the particles, but below that of the matrix, and stretched afterwards (for details we refer to Coertjens et al. ${ }^{27}$, and references therein). 
The particles need to be colloidally stable and of high fluorescent intensity to obtain a sufficiently high photon count during the high speed confocal measurements. Spherical latex particles where synthesised, with the fluorophore covalently bound to the particles. Some experiments were also carried out using particles obtained commercially from Sulfate Microspheres (Molecular Probes, Invitrogen). The latter particles were not suited for high-speed measurements as they lost too much fluorescence intensity during the stretching protocol.

The particle synthesis is adapted from a procedure by Zhang et al. ${ }^{35}$ in which a charged stabilizer is integrated into the polymer chain using a one-step dispersion polymerisation. In a first step $16 \mathrm{~g}$ of styrene monomer (SM) (Sigma Aldrich, 99\%, extra pure), the tertbutylcatechol inhibitor is removed using a pre-packed column. The monomer is then mixed with $165 \mathrm{mg}$ of 4-styrene sulfonate (NaSS, $\mathrm{C}_{8} \mathrm{H}_{7} \mathrm{NaO}_{3} \mathrm{~S}$ ) (Sigma Aldrich) which will act as a charge stabilizer, with $247.4 \mathrm{mg}$ of the initiator, $\alpha, \alpha^{\prime}$ azobisisobutyronitrile (AIBN) (Fluka), in $100 \mathrm{ml}$ of an ethanol-water solution (20 vol\% water at room temperature) and bubbled with $\mathrm{N}_{2}$ to remove oxygen. Half of the mixture is mixed with $40 \mathrm{mg}$ of the fluorescent dye, Pennsylvania green styrene (PGS), the other part is bubbled with $\mathrm{N}_{2}$. After 30 minutes of blanketing, the unlabelled part is added to a heated reactor vessel at $70^{\circ} \mathrm{C}$ and stirred at $200 \mathrm{rpm}$ under a $\mathrm{N}_{2}$ blanket. After an additional 15 min the dyed part is slowly added to the vessel. The reason for the two step procedure is to avoid possible effects of the dye on the particle nucleation. The reaction is then continued for a total reaction time of 8 hours. Pennsylvania green has been selected as it has its excitation peak at $488 \mathrm{~nm}$ which was a suitable laser line and because it has a high stability with strong luminescence. The resulting particles are visualised by SEM and shown in Figure 1. The particle size is observed to be slightly larger than in the one-step synthesis without fluorphore by Zhang et al. ${ }^{35}$.

The PS particles are then embedded in a polyvinylalcohol (PVA) matrix and heated above their melting temperature, but below that of the matrix, and stretched afterwards. After cooling to room temperature, the matrix is degraded and the particles are suspended in 


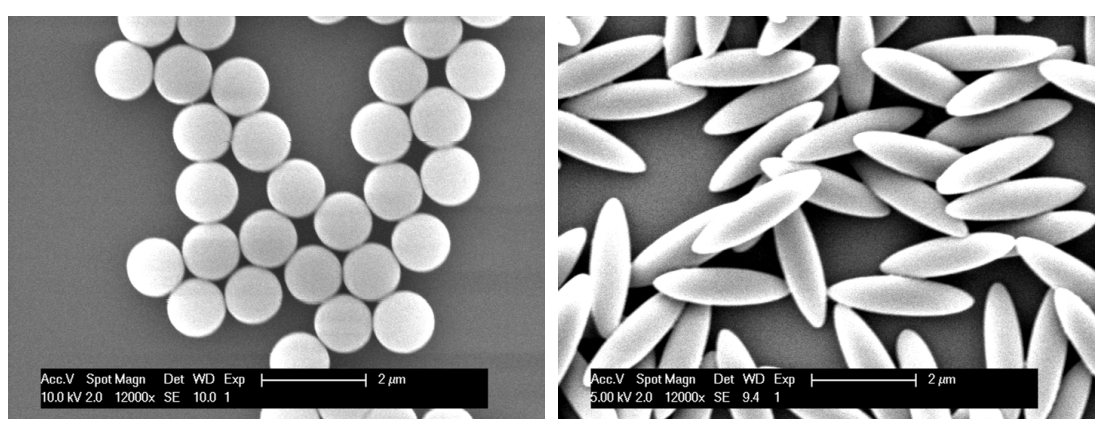

Figure 1. SEM (a) spherical polystyrene spheres of $1.16 \pm 0.04 \mu \mathrm{m}$ and b) typical image of the PS-PG ellipsoids with $A R \approx 3.9$.

the desired medium. The PVA matrix is prepared using 7.5 g PVA (40-88, Sigma-Aldrich) in $300 \mathrm{~g}$ of Milli-Q water (conductivity: $0.055 \mu \mathrm{S}$ ). After dissolution, the mixture is poured over a glass plate $\left(30 \times 30 \mathrm{~cm}^{2}\right)$. After the material has solidified (2 to 3 days) a thin film remains containing the particles. The stretching of the film is performed inside an oil bath at $145^{\circ} \mathrm{C}$ at a speed of $5 \mathrm{~mm} / \mathrm{s}$ (see Coertjens et al. ${ }^{27}$ for details). After cooling, the film strips are washed six times in isopropylalcohol (IPA) and then degraded in an IPA-water mixture (9 $\mathrm{ml}$ IPA for $21 \mathrm{ml}$ water). The PVA is thoroughly removed by three cycles of centrifugation and decanting with an IPA-water mixture with the same IPA to water ratio as before. The presence of residual PVA could be inferred from high resolution SEM images and the observation that aggregates were present in the dispersion. To remove all remaining PVA residues from the particle surface, an additional heating step at $50^{\circ} \mathrm{C}$ is needed, after which one more centrifugation cycle is conducted (see the details of the double cleaning protocol and characterisation methods in Coertjens et al. ${ }^{27}$ ). Finally, one last cycle is executed with pure Milli-Q water. In the end, the particles are suspended in Milli-Q water.

Several batches of ellipsoids with different aspect ratios are prepared. Table 1 contains the results of the analysis of the SEM measurements together with an example in Figure 1. Zeta potential measurements were carried out on these particles using a BIC 90Plus Particle Size S4 Analyzer (Brookhaven) using a $659 \mathrm{~nm}$ laser at $183^{\circ}$, using an electrolyte concentration $1 \mathrm{mM} \mathrm{KCl}$. Typical values ranged from -30 to - 40mV for the particles in reftab:particlespecSEM. 
Table 1. Features of the ellipsoidal particles stretched from both commercial ${ }^{a}$ and home-made fluorescent PS spheres, with $a$ and $b$ the major and minor semi-axis of the particle respectively (averages 20-40 particles).

\begin{tabular}{l|c|c|c|c} 
Particle type & Expected $A R$ & $2 a[\mathrm{~nm}]$ & $2 b[\mathrm{~nm}]$ & $A R(=a / b)[-]$ \\
\hline PS-YG $^{a}$ & $\approx 4.0$ & $2402 \pm 100$ & $604 \pm 25$ & $4.0 \pm 0.2$ \\
PS-PG & $\approx 3.5$ & $2763 \pm 150$ & $760 \pm 30$ & $3.6 \pm 0.2$ \\
PS-PG & $\approx 4.0$ & $2897 \pm 200$ & $735 \pm 50$ & $3.9 \pm 0.2$ \\
PS-PG & $\approx 4.5$ & $3105 \pm 200$ & $722 \pm 40$ & $4.3 \pm 0.2$ \\
PS-PG & $\approx 5.0$ & $3334 \pm 200$ & $683 \pm 25$ & $4.9 \pm 0.3$ \\
\hline
\end{tabular}

${ }^{a}$ FluoSpheres ${ }^{\circledR}$ yellow green Sulfate Microspheres (Molecular Probes, Invitrogen), $1.0 \mu \mathrm{m}$; excitation/emission peak: 505/515 nm; charge: $0.017 \mathrm{meq} / \mathrm{g}$

Fluids used during the experiments include Milli-Q grade water, glycerol (99+\%, Sigma Aldrich), hexadecane (99+\%, Acros Organics), light mineral oil (Sigma Aldrich) and heavy mineral oil (Sigma Aldrich). Before use, all oil phases are purified over an $\mathrm{Al}_{2} \mathrm{O}_{3}$ column (acidic, $50-200 \mu \mathrm{m}$ ) to remove any polar impurities. A slight density mismatch between particle $\left(\rho=1.04 \mathrm{~g} / \mathrm{cm}^{3}\right)$ and sub phase $\left(\rho=1.15 \mathrm{~g} / \mathrm{cm}^{3}\right.$ for a $59 \mathrm{wt} \%$ glycerol-water solution) ensures a very small upward buoyancy force guiding the particle towards the interface over time.

\subsection{Experimental setup}

CryoSEM was used following an approach previously employed to measure the equilibrium contact angles of the ellipsoids ${ }^{27,36}$, with the difference that the samples were not allowed to come to equilibrium. Two small copper moulds are used, one made hollow the other flat. One mould is made hydrophilic by putting it in an oxygen plasma chamber for a few minutes; the other mould remains untreated. The mould is filled with a $0.5 \mu$ l drop of the water phase containing the particles at a concentration of about $1 \%$ wt. On the second mould a $0.5 \mu \mathrm{l}$ drop of the desired oil phase is applied. Both moulds are then pressed together, which creates an oil-water interface and which is left in contact for a few seconds before immobilization. Next, the sample is vitrified using a liquid propane jet (Bal-Tec/Leica JFD 
030) at a temperature of $-120^{\circ} \mathrm{C}$ and fractured under high vacuum at $-110^{\circ} \mathrm{C}$. The sample is then partially freeze-dried for 3 min to remove residual water from the surface. The fracture surface is imaged using a scanning electron microscope in cryogenic mode (Zeiss Gemini 1530). Because the samples are quasi instantaneously vitrified ( $\leq 1 \mathrm{~ms})$, and samples are prepared only seconds after preparing the interface, it is possible to catch certain particles in the act during an adsorption event at the interface.

In microscopy experiments an Olympus IX-71 inverted microscope combined with a VTHawk $^{\mathrm{TM}}$ multi array confocal scan head (VisiTech international) was used yielding a maximum scan rate of 1000 frames per second. To enable three-dimensional imaging, a piezoelectric actuator is installed below the objective with a maximal vertical scanning speed of $20 \mathrm{~ms}$ per frame. The setup is equipped with a dual camera system. An EM-CCD camera (Hamamatsu) allows for high-resolution imaging in three-dimensions with a medium time resolution of 33 frames per second at full frame rate (without binning). To achieve higher frame rates, a cMOS camera (Photron FastCam SA-4) is used in combination with a gated intensified image intensifier (HiCATT, Lambert Instruments), to compensate for the lower quantum efficiency of the cMOS camera. The use of an intensifier lowers the attainable spatial resolution a bit. In practice for the particle used here a frame rate of 125 fps gave good quality images for all sample depths within the objective's working distance. 250 fps could only be maintained for our particles in the immediate vicinity of the bottom plate $(<20-50 \mu \mathrm{m}$ for non-spherical particles, $<\sim 100 \mu \mathrm{m}$ for spheres). In all experiments a 100x UPlanSApo oil immersion objective (Olympus) with a numerical aperture of 1.4 was used in combination with a $488 \mathrm{~nm} 50 \mathrm{~mW}$ He-Ne laser.

The high numerical aperture objective, needed to achieve the required high spatial resolution, is characterised by a very small working distance $(<130 \mu \mathrm{m}$ for the 100x UPlanSApo). Following Park and Furst ${ }^{37}$ we manufactured a miniaturised sample consisting of two borosil- 
icate cylinders. The outer cylinder is made hydrophilic by first cleaning in acetone for 30 minutes using a sonicator to remove organic impurities, and afterwards immersed in a $1 \mathrm{M}$ $\mathrm{NaOH}$ solution to form $\mathrm{SiOH}$-groups on the glass surface. The inner part is silanized using a solution of 5 wt\% dichlorodimethylsilane in n-heptane (Fluka, Sigma Aldrich) for 24 hours. A bottom coverslip (thickness $\mathrm{n}^{\circ} 0$ ) undergoes the same treatment as the outer cylinder to achieve very small contact angles and thus a very thin water layer on top. A 50 micrometer spacer separates the coverslip from the cylinders, and another coverslip at the top seals the environment to avoid fluid evaporation. This suppresses convection and extends the useful measuring time. This sealing is especially necessary to block the capillary gap between the two cylinders, which would drain the trough from its contents even more rapidly than just by evaporation. The exact dimensions of our design can be found in the SI. The sample cells ensure a sharp pinning of the interface with a planar interface being formed.

\subsection{Tracking in two and three dimensions}

The 3D tracking algorithm starts from the well known Crocker-Grier algorithm $^{38}$, which (using Gaussian blurring with a $\sigma$-distance of approximately $0.6-1.0$ ) leads to subpixel accuracy. After finding the center of the object, a region growing procedure is applied to smoothen the particle edges and subsequently a prolate ellipsoid is fit to the object. This fitting then results particle dimensions and orientation angles in every time step. The different time steps are linked together using a simple maximum travel distance constraint, which works well for dilute samples in which particles do not touch each other or seemingly overlap in vertical direction, due to the point spread function (PSF). Generally, multi-array systems use microlenses which are characterised by a rather broad PSF. Deconvolution of the PSF to compensate for the reduced z-resolution, has only limited usefulness in the present experiments due to the use of a microlens array. 
Ideally, one would like to acquire the entire adsorption trajectories, with both the vertical position (z-coordinate) and the altitudinal orientation angle $\varphi$. The latter is defined as the angle between the particle long axis and the interface as illustrated by Figure 2a. However, as it will be shown later it was not possible to perform the z-scanning with sufficient time resolution, due to limitations of even a fast scanning piezo. Therefore, we resorted to a $2 \mathrm{D}$ method, where by using a large confocal pinhole (of $50 \mu \mathrm{m}$ ), the focal depth is increased to image the entire region near the interface. In this manner we can use the maximum scanning speed of the scan head in a plane, but one has to sacrifice the 3D information and is left with a 2D projection of the particle. The altitudinal orientation angle can be obtained from equation 2 .

$$
\varphi=\sin ^{-1}\left(\frac{A R_{\max }-A R}{A R_{\max }-A R_{\min }}\right)
$$

The height information is lost, and can only be inferred from the evolution of $\varphi$ in comparing the results with simulations. In eq. $2 A R_{\max }$ is the maximally observable aspect ratio corresponding to $\varphi=0^{\circ}$ when the particle lies flat, and $A R=1=A R_{\min }$ being the minimum for when the ellipsoid had $\varphi=90^{\circ}$. As the particles are monodisperse, $A R_{\max }$ is known from the SEM characterisation. This method works well, but is numerically not very stable in the vicinity of $90^{\circ}$, and deviations of $5-10^{\circ}$ are expected. However, for angles smaller than $70^{\circ}$ this is no longer an issue, and accuracy is only limited by image quality and the tracking algorithm. Since two-dimensional imaging speed is only limited by decreasing fluorescence intensity with increasing frame rate, this method allows to capture and track the adsorption process at 125 tot 500 frames per second with sufficient accuracy. 


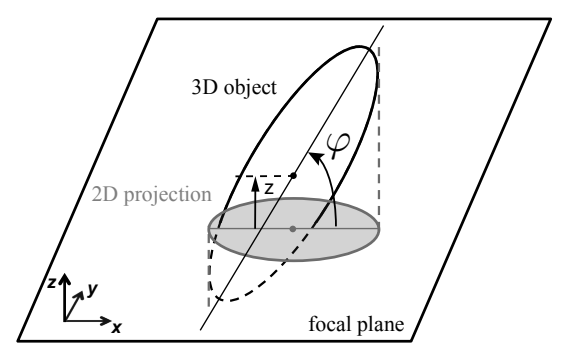

Figure 2. Definition of the trajectory parameters $z$ and $\varphi$ and illustration of the two-dimensional projection.

\section{Results and Discussion}

\subsection{CryoSEM measurements}

Freeze-fracture cryo-SEM was used to qualitatively trace particle trajectories as they enter the interface. Figure 3 shows some typical images in which ellipsoids are "caught in the act" of adsorption, for non-fluorescent PS particles of AR 4 and 6 (particles described in Coertjens et al. ${ }^{?}$. Although it is not possible to accurately quantify the orientation from these pictures because the dimensions of each individual particle would need to be known, it can be seen that the angle of attack, i.e. the altitudinal orientation angle at which the particles penetrate the interface, is typically smaller than $90^{\circ}$, we did not find any particles sticking out high above the interface.

This qualitative observations need to be accompanied by quantitative trajectories. Ideally, to fully map an adsorption trajectory the $(x, y, z)$-coordinates of the particle center during the process are measured along with the orientation. The z- position is challenging. Due to background noise and the field of depth in ordinary microscopy it is difficult to get an accurate result for the vertical position of a moving particle. Using confocal scanning with fast $\mathrm{z}$ scanning, a series of three-dimensional images is acquired over time, enabling complete visualisation of the adsorption process. However, the piezo-electric crystal has a 


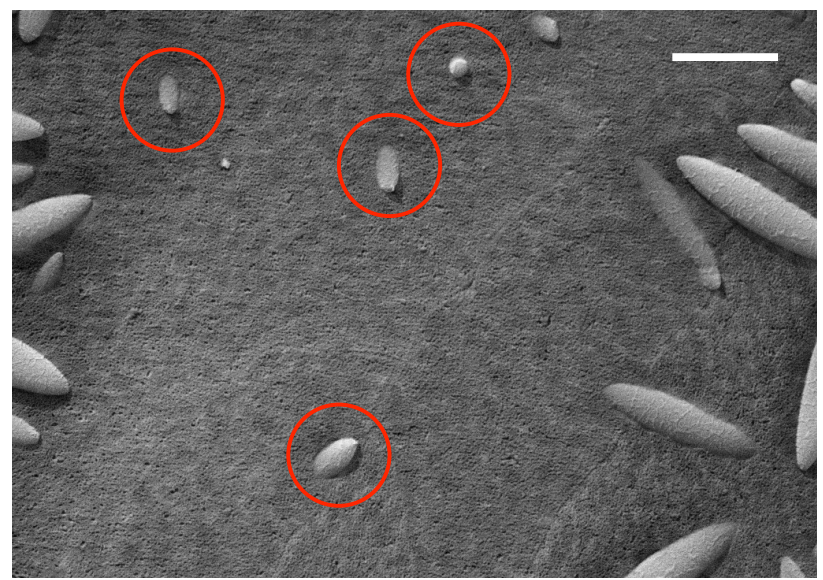

(a)

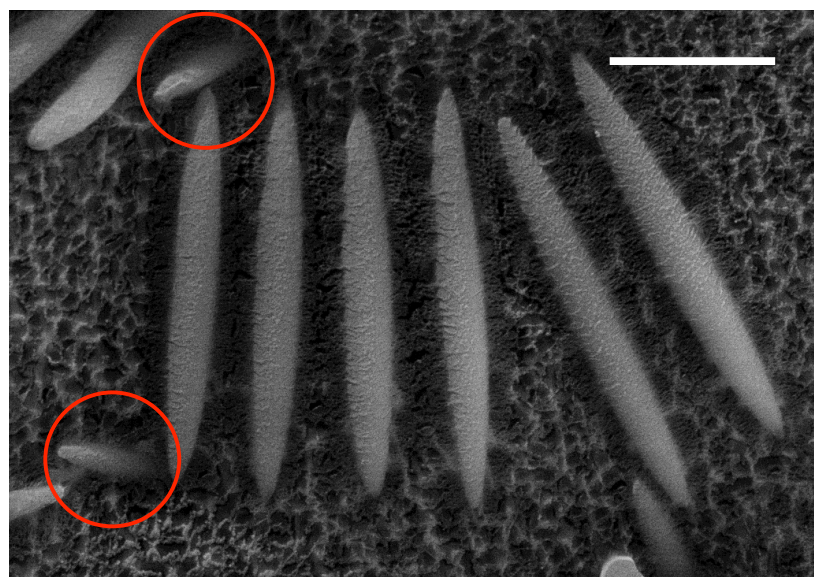

(b)

Figure 3. Cryo-SEM images of PS ellipsoids captured during adsorption (encircled) at an oilwater interface: (a) $A R \approx 4$, (b) $A R \approx 6$. (scale bar: $1 \mu \mathrm{m}$ )

finite response time which leads to a delay of $20 \mathrm{~ms}$ between each slice. To slow down the particle dynamics, the medium viscosity is increased to $\sim 9 \mathrm{mPa} \cdot \mathrm{s}$ using the addition of 59 wt\% of glycerol to the water phase. This allows imaging of particles moving in 3D without too strong image deformations. When imaging a single particle, a reference is helpful to determine the position of the interface. Although the interface is pinned, depending on radial position within the observation cell and unavoidable minor sample volume changes, the height of the local interface varies among experiments. To solve this, particles are always imaged in the same field of view in presence of a particle that is already present at the interface, the latter being identified by their planar configuration.

In Figure 4, a particle initially diffusing in the bulk of the sub phase is imaged and tracked in time and space. As long as the particle remains in the bulk, its trajectory assumes a random walk with a preferred translation direction according to the long axis. The slight buoyancy (Bond numer order $10^{-5}$ ) brings the particles to the interface. At a certain point, the particle suddenly gets pulled into the interface and assumes a horizontal equilibrium position. Even though dynamics are slowed down by the viscosity increase, the adsorption process happens over a time window of the same order as a few time steps of 


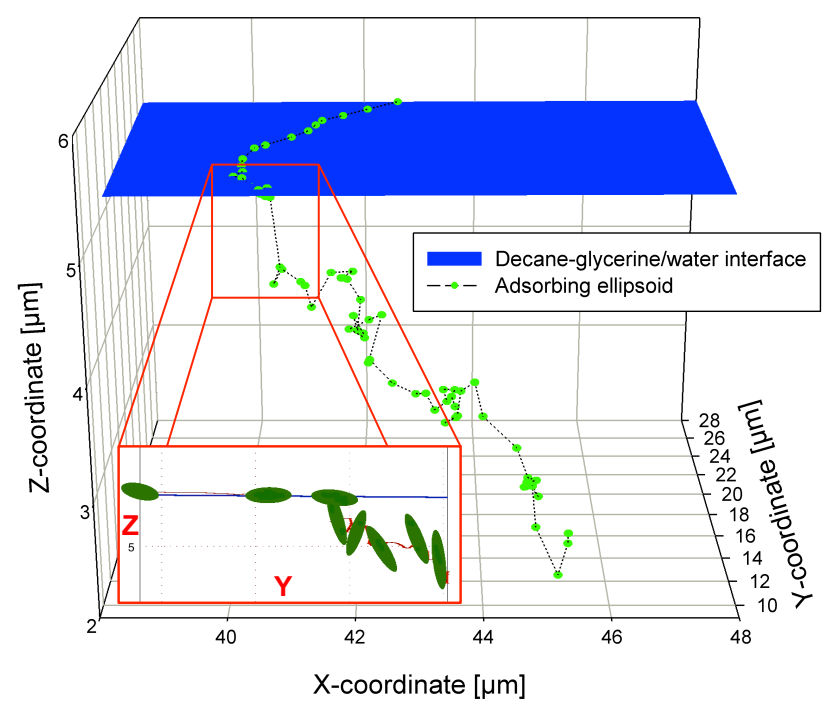

Figure 4. Three-dimensional tracking of a PS ellipsoid (stretched from yellow-green fluorescent FluoSpheres ${ }^{\circledR}$, Invitrogen). Tracks of the particle center from image analysis with the inset showing the $\mathrm{y}$-z side view from which the particle orientation $(\varphi)$ is determinable.

the confocal experiment. Therefore, the vertical position and orientation during the actual adsorption process cannot be determined accurately, only the behaviour right before and after the event can be captured with this method. We can estimate the total adsorption time and the altitudinal orientation angle at the very beginning of adsorption. For a 59 wt\% (10 mPa.s) glycerol-water sub phase and an n-decane top phase, the adsorption process takes a few hundred milliseconds and the altitudinal orientation angle at which the particle touches the interface is $60-70^{\circ}$, in any case lower than $90^{\circ}$.

To obtain better time resolution the near two-dimensional method is applied, which exploits the changes in orientation near the interface, as described in the Materials and Methods section. A typical sequence during adsorption is shown in Figure 5 which are still images from a movie sequence available as supporting information (SI). The image sequence starts with a first reference particle laying flat at the interface, which provides a reference for the position, and a second particle coming into the field of view by diffusion. At first, this second particle moves around by Brownian motion close to the interface (and random orientations are observed, a single snapshot was extracted from the movie and is shown in Figure 5.a). 

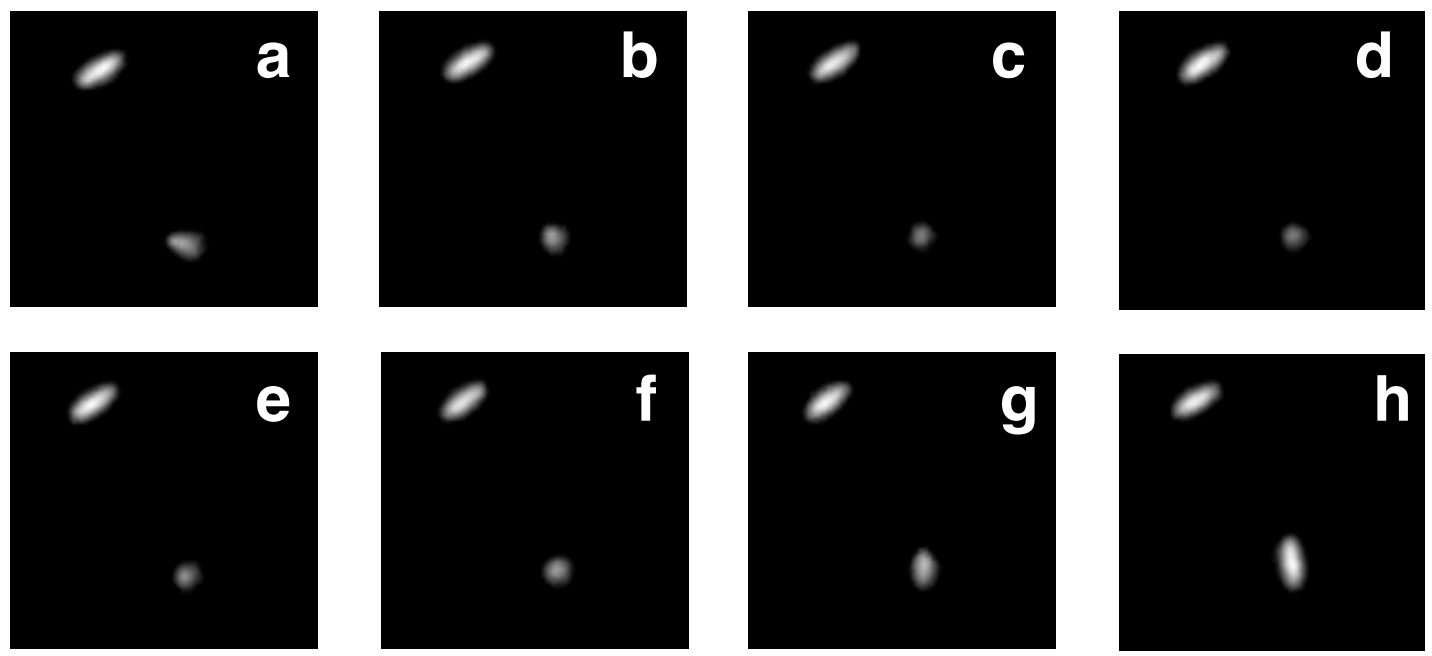

Figure 5. Image sequence during adsorption, a) before adsorption, particle diffusing near interface $(\mathrm{t}=-3 \mathrm{~s}), \mathrm{b})$ particle touches the interface $(\mathrm{t}=0 \mathrm{~s}), \mathrm{c})$ particle moving up $(\mathrm{t}=0.100 \mathrm{~s}) \mathrm{d})$ particle moving up $(t=0.148 \mathrm{~s}), \mathrm{e})$ onset of rotation $(\mathrm{t}=0.200 \mathrm{~s}), \mathrm{f})$ rotation $(\mathrm{t}=0.300 \mathrm{~s}) \mathrm{g})$ rotation $(\mathrm{t}=0.400$ s) h) planar configuration end of fast adsorption $(t=0.700 \mathrm{~s})$

It then touches the interface and subsequently its orientation remains constant for a about 200 milliseconds (Figure $5 \mathrm{~b}-\mathrm{d}$ ), before it starts to rotate, which is shown in panels e-g the particle both end up in a planar configuration Figure $5 \mathrm{~h}$. The whole process takes about half a second. The evolution of the particles' altitudinal orientation angle as a function of time can then be quantified from the images as a function of time.

Figure 6 shows a typical example of the evolution of the altitudinal angle $\varphi$ of an ellipsoidal particle versus time. This specific data set was obtained for a particle with an aspect ratio of 4 and viscosity of $20 \mathrm{mPa} \cdot \mathrm{s}$ on both sides of the interface, and an experiment was selected where the initial angle of attack is large. Four stages can be identified in Figure 6 . In the first zone, the particle is diffusing in bulk. Given the particle dimensions and medium viscosity, the rotational diffusivity in bulk can be estimated from

$$
D_{r}=\frac{\mathrm{k}_{B} T(\ln p+C)}{\pi \eta L^{3}}
$$

where $\eta$ is the medium viscosity, $L$ the particle length, $p$ the aspect ratio, $\mathrm{k}_{B}$ the Boltzman 


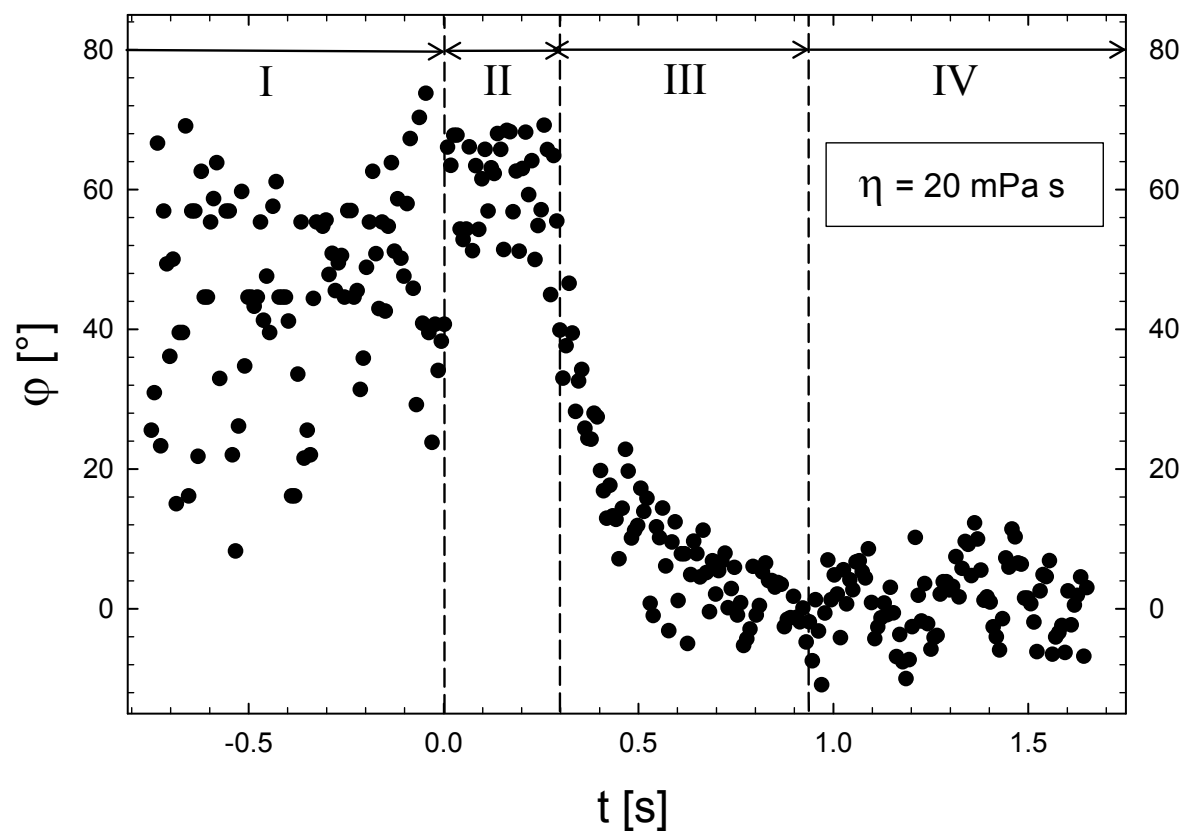

Figure 6. Typical example of the evolution of the altitudinal orientation angle of a prolate PS ellipsoid during adsorption at a liquid-liquid interface $(A R=4, \eta=20 \mathrm{mPas}$, viscosity ratio 1$)$ The distinguishable stages during the process are (I) free diffusion, (II) "vertical" penetration, (III) rotation, (IV) fully adsorbed state. (frame rate: $125 \mathrm{fps}$ )

constant and $C$ a correction factor for finite aspect ratios and hydrodynamic end effects ${ }^{39}$. We find $D_{r}$ to be $\sim 4 \cdot 10^{-3} \frac{\mathrm{rad}}{\mathrm{s}}$ for particles with aspect ratios between 4 and 5 and the viscosity of water, implying that at the sampling speed use here, random jumps in orientation are expected and indeed detected.

In a second stage (II) the particles takes on a fixed altitudinal orientation for a short but distinguishable period of time. This can be expected to correspond to a penetration of the interface, as observed in the cryo-SEM images. Afterwards, in a third stage, it starts rotating towards $0^{\circ}$. The final stage is where the adsorption is complete and out-of-plane orientation no longer changes. In a first set of experiments, the viscosity of both phases is varied by a factor 2 but the viscosity ratio is kept constant. Three closely related viscosities $(\eta=10-15-20 \mathrm{mPa} \cdot \mathrm{s})$ could be used while maintaining the viscosity ratio at 1 , the particle aspect ratio varied between $A R=3.6$ - 4.0 - 4.9. The useful observables are the initial 
Table 2. Fluid properties used in the experiments. $C_{m, g}$ : mass concentration of glycerol in water; $C_{m, h}$ : mass concentration of hexadecane in light mineral oil (or heavy mineral oil in case of Oil150); $\rho_{m i x}$ : density of the fluid mixture; $\eta_{m i x}$ : dynamic viscosity of the fluid mixture.

\begin{tabular}{lcccc} 
Name & $C_{m, g}[\%]$ & $C_{m, h}[\%]$ & $\rho_{m i x}\left[\mathrm{~g} / \mathrm{cm}^{3}\right]$ & $\eta_{m i x}[\mathrm{mPa} \cdot \mathrm{s}]$ \\
\hline Water10 & 61.26 & - & $1.159^{a}$ & $10.00^{a}$ \\
Water15 & 67.05 & - & $1.174^{a}$ & $15.04^{a}$ \\
Water20 & 70.62 & - & $1.184^{a}$ & $19.92^{a}$ \\
Water500 & 93.93 & - & $1.249^{a}$ & $501.6^{a}$ \\
Oil3 & - & 100 & $0.773^{b}$ & 3.0 \\
Oil10 & - & 34 & $0.8194^{c}$ & $10.1^{d}$ \\
Oil15 & - & 18 & $0.8348^{c}$ & $15.2^{d}$ \\
Oil20 & - & 9 & $0.8417^{c}$ & $20.0^{d}$ \\
Oil150 & - & 0 & $0.8669^{c}$ & $145.8^{d}$ \\
\hline${ }^{a}$ calculated from Cheng ${ }^{40}$ for $T=24^{\circ} \mathrm{C}\left(\right.$ Water500: $T=18^{\circ} \mathrm{C}$ ) \\
${ }^{b}$ provided by manufacturer \\
${ }^{c}$ measured at $24^{\circ} \mathrm{C}$ using a 25 ml picnometer \\
${ }^{d}$ measured at $24^{\circ} \mathrm{C}$ using an automated Ubbelohde viscometer (Lauda)
\end{tabular}

altitudinal orientation angle, the time scale of stage II and III and the overall adsorption time. In the second set of experiments, a large viscosity ratio was used and the adsorption trajectories showed a more complex evolution. Table 2 contains all relevant information about the fluid properties for every situation under the experimental environment conditions.

The presence of a region where in the evolution of the altitudinal orientation angle is slow, identified as stage II in Figure 6, means that before adsorbing, each particle positions itself choosing an "angle of attack", which is the altitudinal orientation angle that the particle assumes at the first contact with the interface and before rotation sets in. Comparing this observation of a region of constant initial angle with the calculations of De Graaf et al. ${ }^{31}$ and Gunther et al. ${ }^{32}$, which identify the capillary force as the driving force for adsorption, suggests that the particle penetrates the interface before reorienting, as this pathway corresponds to the fastest changes in free energy. However, there are some significant differences with the simulations. In Figure 7a the initial angle of attack for five to ten adsorption trajectories are shown as a function of AR. It can be observed that in approximately $90 \%$ of all cases this angle of attack $\varphi_{0}$ is found in the range $35-60^{\circ}$, far different from the $90^{\circ}$ 


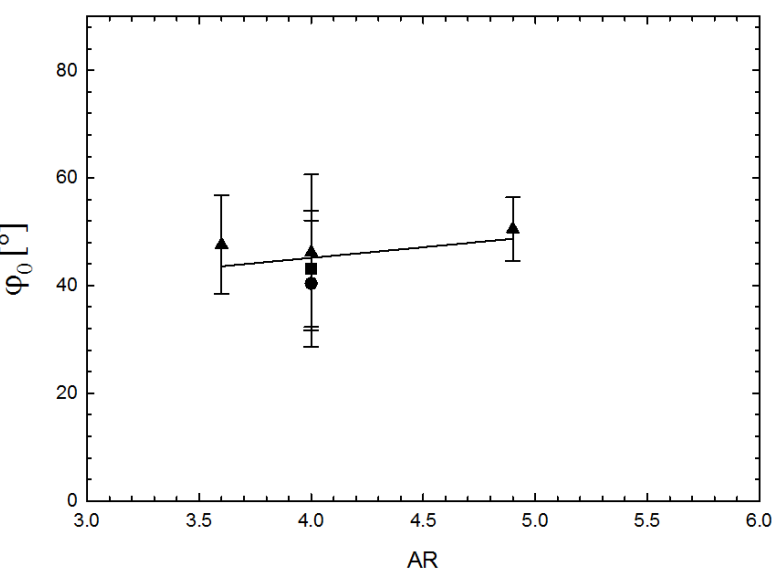

(a)

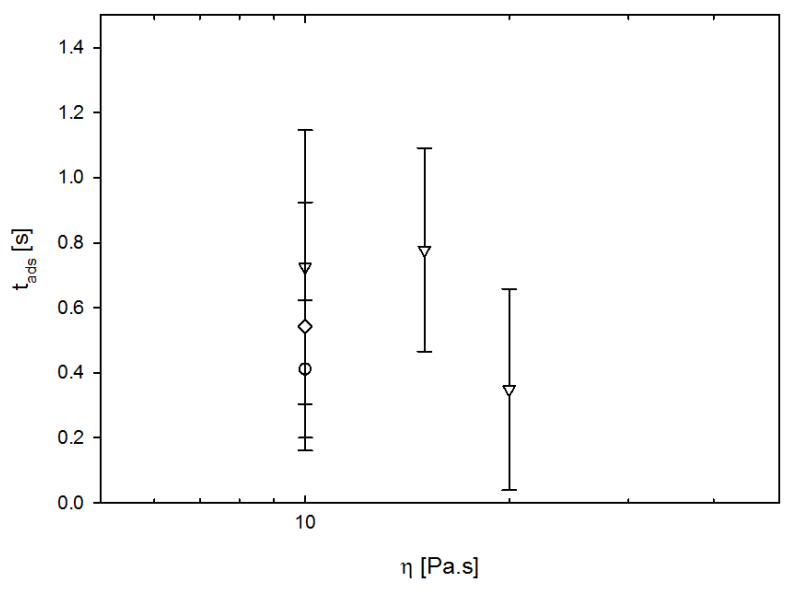

Figure 7. Overview of (a) the "angle of attack" $\varphi_{0}$ as a function of aspect ratio $(\boldsymbol{\Lambda}=10 \mathrm{mPa} \cdot \mathrm{s}$, - $=15 \mathrm{mPa} \cdot \mathrm{s}, \boldsymbol{\square}=25 \mathrm{mPa} \cdot \mathrm{s})(\mathrm{b})$ the adsorption time $t_{a d s}$ as a function of $\eta$ for different values of $A R: 3.6(\bigcirc)-4.0(\nabla)$ and $4.9(\diamond)$.

suggested from the simulations ${ }^{31,32}$. Also, the presence of an undershoot in evolution of the altitudinal orientational angle with time - which was observed in the simulations of both De Graaf et al. ${ }^{31}$ and Gunther et al. ${ }^{32}$, could not be resolved experimentally. So, although the initial entry to the interface must be driven by capillary forces, there are substantial deviations observed, already at short time scales.

After penetrating the interface by a certain distance, stage III begins and particle rotation clearly kicks in and a continuous evolution of the altitudinal angle is observed, towards a planar configuration. At the end of stage III the particle is considered to be adsorbed, 
although slow ageing of the contact line (as in Coertjens et al. ${ }^{27}$ ) may still occur. Data points in this region IV however are a bit more noisy $\left( \pm 10^{\circ}\right)$ as convection can draw the particle away from the center of the field of view and small effects of curvature may also be present, limiting our accuracy over long time scales.

For systems very close to the experimental systems studied here, De Graaf et al. ${ }^{31}$ obtained the adsorption times under the assumptions that capillary and viscous forces were the only ones at play. For a particle of aspect ratio 6 , a viscosity of $1 \mathrm{mPa} \cdot \mathrm{s}$ and a surface tension of $10 \mathrm{mN} / \mathrm{m}$ they predict an adsorption time of order $10^{-3} \mathrm{~ms}$. When only capillary and viscous forces are present the time scale is expected to be proportional to the viscosity (decreasing to $10^{-2} \mathrm{~ms}$ for $10 \mathrm{mPa} \cdot \mathrm{s}$ ) and inversely proportional to the interfacial tension. As can be clearly seen from Figure 6, the timescales observed here are orders of magnitude longer, rather several 100 milliseconds up to a second, or four orders of magnitude slower. The simulations by De Graaf et al. ${ }^{31}$ also predict the timescale for the initial entry is predicted to be $25 \%$ longer compared to the timescale for the subsequent reorientation, as the change in area which governs the capillary driving force is expected to increase as the particle start to reorient. Experimentally, we typically observe a timescale for the constant altitudinal orientation angle of order $200 \mathrm{~ms}$ and the reorientation takes two to three times as long. So whereas some features of a capillary driven adsorption are maintained, the kinetics and the pathways reveal clear differences. This longer time scale is in congruence with the recent observations of Wang et al. ${ }^{33}$. However, Wang et al. do not resolve the constant angle stage II in their experiments.

To determine the adsorption time in a more carefree and objective manner, the evolution of the orientation angle with time is fitted with a 5-parameter sigmoid, with parameters (a, 
b, c, d and e).

$$
\varphi(t)=a+\frac{b}{\left(1+\exp \left(\frac{-(t-c)}{d}\right)\right)^{e}}
$$

An example of a fit to typical experimental data is shown in figure S.1 (supporting info). Both the horizontal asymptotes to this sigmoid and the tangent through the inflection point are then determined. A characteristic adsorption time $t_{a d s}$ is defined as the time lapse between the two intersection points created by these three straight lines as indicated in the supporting Figure S.2. This characteristic adsorption time is a bit shorter than the actual adsorption time starting from the first contact between particle and interface until equilibrium in a horizontal position (because of the presence of stage II), but is longer than the time required for rotation only.

When examining this adsorption time, Figure $7 \mathrm{~b}$, no significant change is noticed for higher viscosities. Although the viscosity has been increased up to a maximum of a factor two, the the highest viscosity even has a shorter average $t_{a d s}$ than the others, although the experimental error is large. For purely capillary systems the adsorption time should scale inversely with the viscosity ${ }^{31}$, in a contact line dominated regime, Wang et al predict that it should only be determined by particle properties ${ }^{33}$. The experiments in Figure 7 do not enable us to make any definitive statements.

To investigate if there is a role for viscous forces we deliberately applied a strong viscosity gradient over the interface. In a first set of experiments, Water10 and Oil150 mixtures are prepared as sub and top phase with viscosities $\eta_{w}$ and $\eta_{\text {oil }}$ respectively (see Table 2) leading to a viscosity ratio of 15 across the interface. For these experiments, PS-PG ellipsoids with an $A R \approx 4.9$ are used. A typical orientation pathway encountered in this situation is depicted in Figure 8a. The quality of the fit of the sigmoidal function is not as good. The rotation stage is slowed down somewhat compared to the experiments of Figure 7, with 


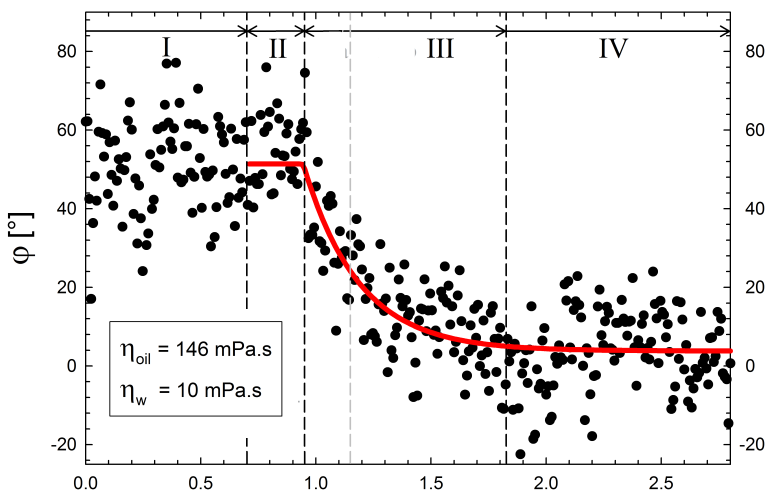

(a)

$\mathrm{t}[\mathrm{s}]$

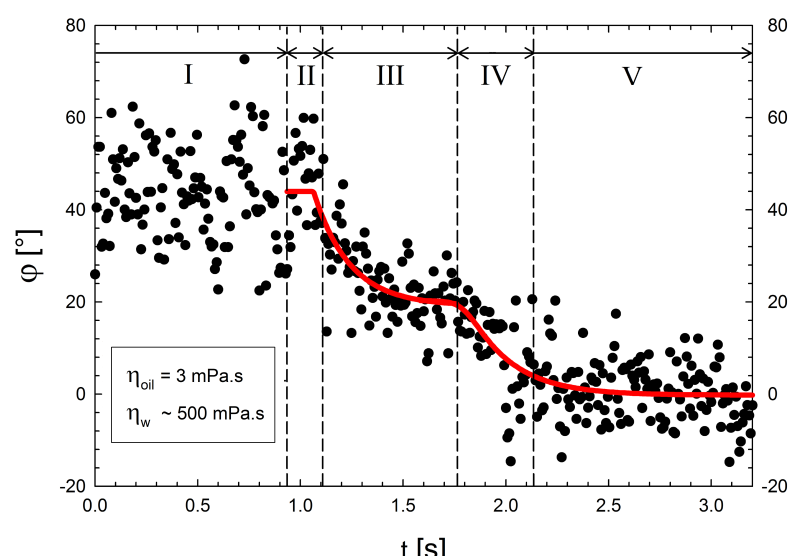

(b)

Figure 8. Altitudinal orientation angle of a prolate PS ellipsoid $(A R=4.9)$ during adsorption at an oil-water interface with a viscosity gradient: (a) $\eta_{o i l} \gg \eta_{w}$, (b) $\eta_{o i l} \ll \eta_{w}$. Time is reported from the strat of the acquisition (125 fps). The lines are drawn by hand to guide the eye.

$\eta \sim 10-20$ mPa.s. However, this is only a factor of about two whereas the viscosity jump is now a factor of 15 . When the situation is inverted, having a more viscous aqueous phase, using Water500 and Oil3 as sub and top phase respectively leading to a viscosity ratio of 0.006, a trajectory as in Figure $8 \mathrm{~b}$ is obtained. The quality of the sigmoidal fit decreases further and in some experiments a shoulder could be observed, i.e. a short delay in rotation during transition to the less viscous phase. Again the adsorption time is increased slightly, by a factor $2 \sim 3$, but a scaling with viscosity - as expected based on capillarity - is not observed.

Regarding the "angle of attack", $\varphi_{0}$, no significant differences are noticed between the two situations or with respect to the data shown in Figure 7, which is in agreement with 
this angle of attack only being dependent on the local particle curvature and fluid-particle wetting properties regardless of viscosity.

The global picture which emerges from the experiments is that the general features of a capillary driven adsorption process are still present, which starts with an initial phase where the altitudinal orientation angle upon penetration of the interface remains constant, followed a second stage characterised by a slow rotation towards a planar configuration. Comparing our results with the predictions of the simulations that take into account capillary forces and viscous friction ${ }^{31,32}$, the initial angles of attack we observe are typically smaller than those seen in the simulations and there is substantial spread on the angle. This could be due to small deviations from an ideal ellipsoidal shape or effects of local contact line pinning near the tips. The constant angle stage was not observed in the recent experiments by Wang et al. ${ }^{33}$, maybe due to the limited time resolution of their experiments and the fact that the particles are pushed towards the interface by an optical trap, which may cause additional tendency for alignment.

The adsorption times are much longer than expected based on the predictions of a Langevin equation which balances capillary forces and viscous friction ${ }^{31}$. This observation is in line with the measurements of Wang et al. ${ }^{33}$, who also report adsorption time scales which are orders of magnitude higher than expected based on capillary and viscous effects alone. Wang et al. ${ }^{33}$ were also able to show a coupling between the rotational and translational motion, as their holographic technique provides them with both observables. Following earlier work, which focussed on the slow evolution of the contact angle ${ }^{25}$, they state that thermal fluctuations drive the net displacement of the contact line, hindered by surface nanostructures. In our experiments we also did not observe a simple scaling of the adsorption time with viscosity . Yet, the experiments with the viscosity mismatch here show that dissipative process may still play a secondary role in the movement of the contact line 
and the reorientation of the particles.

A few final comments concern the role of interface deformations and colloidal forces. In adsorption experiments, the colloidal interactions between the interface (which may carry electrostatic charges) and the charges on the particle may also influence the efficiency of the adsorption ${ }^{22}$, and this was not considered here. In the present work we only consider dynamics once the particle breaches the interface, when capillary energy is expected to become overwhelming. Second, in relation to possible flow-induced interface deformations by single particles, calculating the deformation rates from the observed adsorption times and the dimension of the particles, we can provide a coarse estimate of the Capillary number, on the order of $10^{-3}$. As the experimentally observed adsorption rates are slower than predicted for the case in which only capillary and viscous forces occur, dynamic deformations of the interface by the particles are not very likely. They are also not observed in the cryo-SEM experiments. Of course the constant contact angle requirement may also influence the orientational transition for quantitative calculations of the transition as was shown for magnetic particles $^{42,43}$.

It can be hypothesised that the origin of the contact line pinning lies in small surface heterogeneities. Residual PVA may still be present, although we typically observe this in high resolution SEM images (see Coertjens et $\mathrm{al}^{27}$ ). Charge heterogeneity at a larger scale was investigated by Ho et al. ${ }^{34,44}$ where nanoparticles were shown to adsorb homogeneously to the surface of the particles. However, it is known that the surfactant-free polymerisation of polystyrene may lead to small scale heterogeneities ${ }^{45,46}$, and even for spheres there is substantial heterogeneity in the colloidal interactions between PS spheres at oil water interfaces $^{47}$. Studying phenomena at fluid-fluid interfaces brings out these features which would be obscured in most bulk experiments. 


\section{Conclusions}

The adsorption of ellipsoidal particles to liquid interfaces is characterised by a complex nonmonotonic evolution of the altitudinal orientation angle, suggesting the pathway reflects essential features of adsorption driven by capillarity as the fundamental driving force. However, the adsorption dynamics are orders of magnitude slower than expected based solely on the effects of capillary forces. This confirms the role of surface heterogeneities on the motion of the contact line. The role of contact line hopping alone may not be sufficient to account for the initial stages of adsorption. Further investigations on the adsorption kinetics of anisotropic colloids at fluid interfaces are in demand to elucidate the presence of metastable wetting states ${ }^{48}$, or to shed light on the dynamical evolution of particles with switchable orientations upon application of external fields ${ }^{49,50}$.

\section{Acknowledgements}

Support by the Swiss National Science Foundation (grant 200021-157147) and the Hercules foundation Flanders are kindly acknowledged.

\section{References}

(1) Bresme, F.; Oettel, M., Nanoparticles at fluid interfaces. J. Phys. Cond. Matt., 2007, $19,413101$.

(2) Pieranski P., Two-dimensional interfacial colloidal crystals Phys. Rev. Lett., 1980, 45,569 .

(3) Denkov, N.D. Two-dimensional crystallization, Nature, 1993, 361, 26.

(4) Stancik, E.J.; Gavranovic, G.T.; Widenbrant, M.J.; Laschitsch, A.T.; Vermant, J.; Fuller, G.G., Structure and dynamics of particle monolayers at a liquid-liquid interface subjected to shear flow, Faraday Discussions, 2003, 123, 145. 
(5) Keim, N.C.; Arratia, P.E., Mechanical and Microscopic Properties of the Reversible Plastic Regime in a 2D Jammed Material, Phys. Rev. Lett., 2014, 112, 028302.

(6) Buttinoni, I., Zell, Z.A., Squires, T.M.; Isa, L. Colloidal binary mixtures at fluid-fluid interfaces under steady shear: structural, dynamical and mechanical response, Soft Matter, 2015, 11, 8313.

(7) Stamou, D.; Duschl, C., ; Johannsmann, D., Long-range attraction between colloidal spheres at the air-water interface: The consequence of an irregular meniscus Phys. Rev. E, 2000, 62, 5263 .

(8) Loudet, J. C., Yodh, A. G., ; Pouligny, B., Wetting and contact lines of micrometersized ellipsoids. Phys. Rev. Lett., 2006, 97, 018304.

(9) Lehle, H., Noruzifar, E., ; Oettel, M. Ellipsoidal particles at fluid interfaces, The European Physical Journal E , 2008, 26, 151.

(10) Gonzenbach, U. T., Studart, A. R., Tervoort, E., ; Gauckler, L. J., Ultrastable ParticleStabilized Foams. Angewandte Chemie International Edition, 2006, 45, 3526 .

(11) Binks, B. P.; Murakami, R., Phase inversion of particle-stabilized materials from foams to dry water, Nature materials, 2006 , 5, 865.

(12) Arditty, S.; Schmitt, V.; Lequeux, F.; Leal-Calderon, F. Interfacial Properties in SolidStabilized Emulsions, Eur. Phys. J. B , 2005 , 44, 381.

(13) Cates, M. E., ; Clegg, P. S. Bijels: a new class of soft materials,, 4(11), 2132-2138. Soft Matter, 2008 , 4, 2132.

(14) Imperiali,L., Clasen, Ch., Fransaer, J., Macosko, C.W., Vermant, J., A Simple Route to Graphene Oxide Frameworks, Materials Horizons , 2014, 1, 139. 
(15) Isa, L., Kumar, K., Muller, M., Grolig, J., Textor, M.; Reimhult, E. (2010). Particle lithography from colloidal self-assembly at liquid-liquid interfaces, ACS Nano, 2010, 4, 5665 .

(16) Madivala, B.; Vandebril, S.; Fransaer, J.; Vermant, J., Exploiting Particle Shape in Solid Stabilized Emulsions, Soft Matter, 2009, 5, 1717.

(17) Yunker, P. J., Still, T., Lohr, M. A., ; Yodh, A. G. (2011). Suppression of the coffee-ring effect by shape-dependent capillary interactions, Nature, 2011, 476, 308.

(18) Vermant, J. (2011). Fluid mechanics: When shape matters. Nature, 2011, 476, 286.

(19) Koretsky and P. Kruglyakov, Izv. Sib. Otd. Akad. Nauk USSR , 1971, 139, 19.

(20) Levine, S.; Bowen, B. D.; Partridge, S. J.; Stabilization of emulsions by fine particles I. Partitioning of particles between continuous phase and oil/water interface. Colloids and Surfaces, 1989, 38, 325 .

(21) Du, K., Glogowski, E., Emrick, T., Russell, T. P.; Dinsmore, A. D. Adsorption energy of nano-and microparticles at liquid? liquid interfaces.Langmuir, 2010, 26, 12518.

(22) Dugyala, V. R., Muthukuru, J. S., Mani, E., ; Basavaraj, M. G. (2016). Role of electrostatic interactions in the adsorption kinetics of nanoparticles at fluid-fluid interfaces, Physical Chemistry Chemical Physics, 2016, 18, 5499.

(23) Kaz, D. M., McGorty, R., Mani, M., Brenner, M. P., ; Manoharan, V. N. (2012). Physical ageing of the contact line on colloidal particles at liquid interfaces, Nature materials , 2012, 11, 138.

(24) Colosqui, C. E., Teng, T., Rahmani, A. M. (2015). Wetting Driven by Thermal Fluctuations on Terraced Nanostructures, Phys. Rev. Lett., 2015, 115154504. 
(25) Rahmani A.M.; A. Wang; Manoharan V.N., Colosqui C.N.; Colloidal particle adsorption at liquid interfaces: Capillary driven dynamics and thermally activated kinetics Soft Matter, 2016, 12, 6365.

(26) Boniello, G., Blanc C.,Fedorenko D., Medfai M, Ben Mbarek N., In M., Gross M., Stocco A., Nobili M., Brownian diffusion of a partially wetted colloid Nature materials, 2015, 14, 908 .

(27) Coertjens, S.; Moldenaers, P.; Vermant, J.; Isa, L. Contact Angles of Microellipsoids at Fluid Interfaces. Langmuir 2014, 30, 4289.

(28) Decuzzi, P.; Pasqualini, R.; Arap, W.; Ferrari, M. Intravascular Delivery of Particulate Systems: Does Geometry Really Matter? Pharm. Res., 2008, 26, 235.

(29) Decuzzi, P.; Ferrari, M. The Receptor-Mediated Endocytosis of Nonspherical Particles. Biophys. J. 2008, 94, 3790.

(30) Mihut, A. M.; Dabkowska, A. P.; Crassous, J. J.; Schurtenberger, P.; Nylander, T. Tunable Adsorption of Soft Colloids on Model Biomembranes. ACS Nano 2013, 7, 10752.

(31) de Graaf, J.; Dijkstra, M.; van Roij, R. Adsorption Trajectories and Free-Energy Separatrices for Colloidal Particles in Contact with a Liquid-Liquid Interface, J. Chem. Phys., 2010, 132, 164902.

(32) Günther, F.; Janoschek, F.; Frijters, S.; Harting, J. Lattice Boltzmann Simulations of Anisotropic Particles at Liquid Interfaces, Computers Fluids , 2012, 80, 184.

(33) Wang A., Rogers W.B., Manoharan V.N., Effects of contact-line pinning on the adsorption of nonspherical colloids at liquid interfaces, arXiv:607.08167v1 2016.

(34) Ho, C. C.; Keller, A.; Odell, J. A.; Ottewill, R. H. Preparation of Monodisperse Ellipsoidal Polystyrene Particles, Colloid Polym. Sci. , 1993, 271, 469. 
(35) Zhang, F.; Cao, L.; Yang, W. Preparation of Monodisperse and Anion-Charged Polystyrene Microspheres Stabilized with Polymerizable Sodium Styrene Sulfonate by Dispersion Polymerization. Macromol. Chem. Phys. 2010, 211, 744.

(36) Isa, L.; Lucas, F.; Wepf, R.; Reimhult, E. Measuring Single-Nanoparticle Wetting Properties by Freeze-Fracture Shadow-Casting Cryo-Scanning Electron Microscopy. Nat. Comm. 2011, 2,

(37) Park, B. J.; Furst, E. M. Attractive Interactions Between Colloids at the Oil-Water Interface. Soft Matter 2011, 7, 7676.

(38) Crocker J.C.; Grier, D.G., Methods of digital video microscopy for colloidal studies, J. Coll. Int. Sci., 1996, 179, 298(1996).

(39) Ortega, A.; de la Torre, J. G. Hydrodynamic properties of rodlike and disklike particles in dilute solution, J. Chem. Phys., 2003 , 119, 9914.

(40) Cheng, N.-S. Formula for the Viscosity of a Glycerol-Water Mixture. Ind. Eng. Chem. Res. 2008, 47, 3285.

(41) Miner, C. S. Dalton, N.N, Glycerol American Chemical Society (1953).

(42) Davies, G.B., Krüger, T., Coveney, P.V., Harting, J.; Bresme, F Interface deformations affect the orientation transition of magnetic ellipsoidal particles adsorbed at fluidfluid interfaces, Soft Matter, 2014, 10, 6742 .

(43) Newton, B.J., Brakke A.K., Buzza D.M.A., Influence of magnetic field on the orientation of anisotropic magnetic particles at liquid interfaces, Physical Chemistry Chemical Physics 2014, 16, 26051.

(44) Ho, C. C., M. J. Hill, and J. A. Odell Morphology of ellipsoidal latex particle, Polymer, 1993 34: 2019. 
(45) Feick J.D., D. Velegol, Measurements of charge nonuniformity on polystyrene latex particles. Langmuir, 2002, 18, 3454.

(46) D. Feick, N. Chukwumah, A. E. Noel D. Velegol, Altering surface charge nonuniformity on individual colloidal particles. Langmuir, 2004, 20: 3090,.

(47) Park, B. J., Vermant, J., Furst, E. M., Heterogeneity of the electrostatic repulsion between colloids at the oilwater interface. Soft Matter, 2010, 6, 5327.

(48) Isa, L.; Samudrala, N. ;Dufresne, E.R. Adsorption of sub-micron amphiphilic dumbbells to fluid interfaces. Langmuir, 2014, 30 , 5057

(49) Davies, G.B., Krüger, T., Coveney, P.V., Harting, J.; Bresme, F. Assembling ellipsoidal particles at fluid interfaces using switchable dipolar capillary interactions, Advanced Materials, 2014, 26, 6715 .

(50) Newton, B.J.; Buzza, M.A. Magnetic cylindrical colloids at liquid interfaces exhibit non-volatile switching of their orientation in an external field, Soft Matter, 2016 ,12 , 5285 . 